\title{
Recommendations on Surveillance for Differentiated Thyroid Carcinoma in Children with PTEN Hamartoma Tumor Syndrome
}

\author{
L.A. Jonker ${ }^{a} \quad$ C.A. Lebbink ${ }^{a, b} \quad$ M.C.J. Jongmans ${ }^{b, c}$ R.A.J. Nievelstein ${ }^{b, d}$ \\ J.H.M. Merks ${ }^{b}$ E.J.M. Nieveen van Dijkum ${ }^{\text {e }} \quad$ T.P. Links $^{f} \quad$ N. Hoogerbrugge ${ }^{g}$ \\ A.S.P. van Trotsenburg ${ }^{h}$ H.M. van Santen ${ }^{a, b}$ \\ a Department of Pediatric Endocrinology, Wilhelmina Children's Hospital, University Medical Center Utrecht, Utrecht, \\ The Netherlands; ${ }^{b}$ Princess Máxima Center for Pediatric Oncology, Utrecht, The Netherlands; ' ${ }^{\circ}$ epartment of Genetics, \\ University Medical Center Utrecht, Utrecht, The Netherlands; ${ }^{\mathrm{d}}$ Department of Pediatric Radiology and Nuclear \\ Medicine, Wilhelmina Children's Hospital, University Medical Center Utrecht, Utrecht, The Netherlands; ${ }^{\mathrm{e} D e p a r t m e n t}$ of \\ Surgery, Cancer Center Amsterdam, Amsterdam University Medical Center, Amsterdam, The Netherlands; ${ }^{\mathrm{f} D e p a r t m e n t}$ \\ of Endocrinology, University Medical Center Groningen, Groningen, The Netherlands; ${ }^{9}$ Department of Human Genetics,

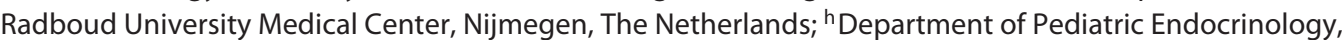 \\ Emma Children's Hospital, Amsterdam University Medical Center, Amsterdam, The Netherlands
}

\section{Keywords}

Differentiated thyroid carcinoma - PTEN hamartoma tumor syndrome, pediatric - Thyroid carcinoma surveillance program $\cdot$ Thyroid cancer genetics

\begin{abstract}
Background: PTEN hamartoma tumor syndrome (PHTS) represents a group of syndromes caused by a mutation in the PTEN gene. Children with a germline PTEN mutation have an increased risk of developing differentiated thyroid carcinoma (DTC). Several guidelines have focused on thyroid surveillance in these children, but studies substantiating these recommendations are lacking. Objective: The present study intends to provide the available evidence for a thyroid carcinoma surveillance program in children with PHTS. Methods: An extensive literature search was performed to identify all studies on DTC in pediatric PHTS patients. Two pediatric cases are presented to illustrate the pros and cons of thyroid carcinoma surveillance. Recommendations for other patient groups at risk for DTC were evaluated. Consensus within the study team on rec-
\end{abstract}

karger@karger.com www.karger.com/etj Karger"
(C) 2020 The Author(s)

Published by S. Karger AG, Basel

Karger

Open access

This article is licensed under the Creative Commons AttributionNonCommercial-NoDerivatives 4.0 International License (CC BYNC-ND) (http://www.karger.com/Services/OpenAccessLicense). Usage and distribution for commercial purposes as well as any distribution of modified material requires written permission. ommendations for children with PHTS was reached by balancing the incidence and behavior of DTC with the pros and cons of thyroid surveillance, and the different surveillance methods. Results: In 5 cohort studies the incidence of DTC in childhood ranged from 4 to $12 \%$. In total 57 cases of DTC and/or benign nodular disease in pediatric PHTS patients were identified, of which 27 had proven DTC, with a median age of 12 years (range 4-17). Follicular thyroid carcinoma (FTC) was diagnosed in $52 \%$ of the pediatric DTC patients. No evidence was found for a different clinical behavior of DTC in PHTS patients compared to sporadic DTC. Conclusions: Children with PHTS are at increased risk for developing DTC, with 4 years being the youngest age reported at presentation and FTC being overrepresented. DTC in pediatric $\mathrm{PHTS}$ patients does not seem to be more aggressive than sporadic DTC. Recommendations: Surveillance for DTC in pediatric PHTS patients seems justified, as early diagnosis may

First and second authors L.A. Jonker and C.A. Lebbink contributed equally to this work.

Senior authors A.S.P. van Trotsenburg and H.M. van Santen contributed equally to this work. 
decrease morbidity. Consensus within the study team was reached to recommend surveillance from the age of 10 years onwards, since at that age the incidence of DTC seems to reach $5 \%$. Surveillance for DTC should consist of yearly neck palpation and triennial thyroid ultrasound. Surveillance in children with PHTS should be performed in a center of excellence for pediatric thyroid disease or PHTS. @ 2020 The Author(s)

Published by S. Karger AG, Basel

\section{Introduction}

The PTEN hamartoma tumor syndrome (PHTS) is the encompassing name for the rare diseases Cowden syndrome, Bannyan-Riley-Ruvalcaba syndrome, and Proteus-like syndrome, and is caused by autosomal dominant mutations in the phosphatase and tensin homolog (PTEN) gene [1]. PHTS is clinically characterized by macrocephaly, developmental delay and hamartomas. In addition, due to the pathogenic variant in PTEN, affected patients have a greatly increased risk to develop malignant neoplasms, including carcinomas of the breast, thyroid gland, endometrium, colon, and kidney [2].

Differentiated thyroid carcinoma (DTC) is one of the common cancer types in patients with PHTS, with a lifetime risk of $35-38 \%$ [2]. This increased risk is already present during childhood, whereas the risk of developing other types of cancer during childhood appears to be equal to that of the general population [2]. This may warrant periodic surveillance for DTC in affected children, and currently several thyroid surveillance recommendations for PHTS patients have been proposed [3-7].

The National Comprehensive Cancer Network (NCCN) in the USA recommends that pediatric PHTS patients receive an annual thyroid ultrasound from the age of diagnosis onwards [3]. In contrast, the American Thyroid Association advises to perform annual physical examinations of the thyroid gland in children from the age of diagnosis of PHTS onwards with additional thyroid ultrasound in case of palpable nodule(s), thyroid asymmetry, or abnormal cervical lymph node(s) [4]. The Dutch association for clinical geneticists (VKGN) proposes annual surveillance of the thyroid gland in children, by means of palpation or thyroid ultrasound. The Dutch VKGN guideline for adult PHTS patients recommends yearly neck palpation, thyroid ultrasound yearly or once every 2 years, and annual serum thyroid-stimulating hormone concentration [6]. The UK Cancer Genetic Group advises to perform annual ultrasound surveillance from the age of 16 years onwards, or earlier in case of a family history for DTC or after informed discussion with the patient and its family [5].

Although these guidelines agree on once a year surveillance frequency, they are discordant with regard to the age at start and the method of surveillance. It may be questioned on which evidence these guidelines are based, considering the scarcity of studies in pediatric PHTS patients.

Consensus within the study team has been reached that surveillance for any childhood cancer may be justified when its incidence reaches 5\%, also for tumors with a good prognosis $[8,9]$. For this reason, the first question that needs to be answered is about the incidence of DTC in children with PHTS.

The second question that needs to be addressed is whether there is evidence that DTC in PHTS behaves differently than sporadic DTC.

Thirdly, surveillance is only justified if its benefit outweighs any possible harm; in other words, surveillance should improve disease outcome. Therefore, the third question that should be answered before formulating recommendations on surveillance is whether detecting DTC in an early stage is beneficial for a child with PHTS.

In addition to a literature search, review, and consensus discussion, we present 2 cases to illustrate the pros and cons of surveillance for DTC in children with PHTS. We aimed to formulate optimal recommendations for surveillance of DTC in children with PHTS based on the best available evidence that also meet the (clinical) needs of young patients.

\section{Methods}

An extensive literature search was done using PubMed and Embase for the age of onset of thyroid abnormalities and benefits of surveillance for DTC in pediatric PHTS patients (Fig. 1). There was no date limit. Articles written in English, German, Dutch, and French were included. Data on children with a clinical diagnosis based on the PHTS criteria as well as on children with a proven PTEN mutation were included in our analysis. We set no minimum threshold for the number of patients in a cohort for studies to be included. Additionally, the references of all qualifying articles were meticulously screened to identify articles that were missed in the initial search.

To illustrate the pros and cons of surveillance for DTC in children with PHTS, 2 cases are presented. Informed consent was obtained from both patients and their parents for publication.

Results of the literature search were discussed within the national expert panel, including: pediatric endocrinologists, endocrinologists, a pediatric radiologist, a pediatric oncologist, a pediatric surgeon, and a clinical geneticist. All panel members have special expertise in pediatric thyroid carcinoma and/or PHTS. Recommendations for surveillance were formulated. 
Fig. 1. Flow diagram of extensive literature results. Search strategy, Pubmed, search terms: (Cowden syndrome OR Cowden disease OR Bannayan Riley Ruvalcaba syndrome OR PTEN hamartoma syndrome) AND (thyroid cancer OR thyroid carcinoma OR thyroid nodule OR thyroid disease). To specify the search for childhood data, the terms (child OR pediatrics) were added. In total, 169 papers were found by the search of which 47 were included in this recommendation.

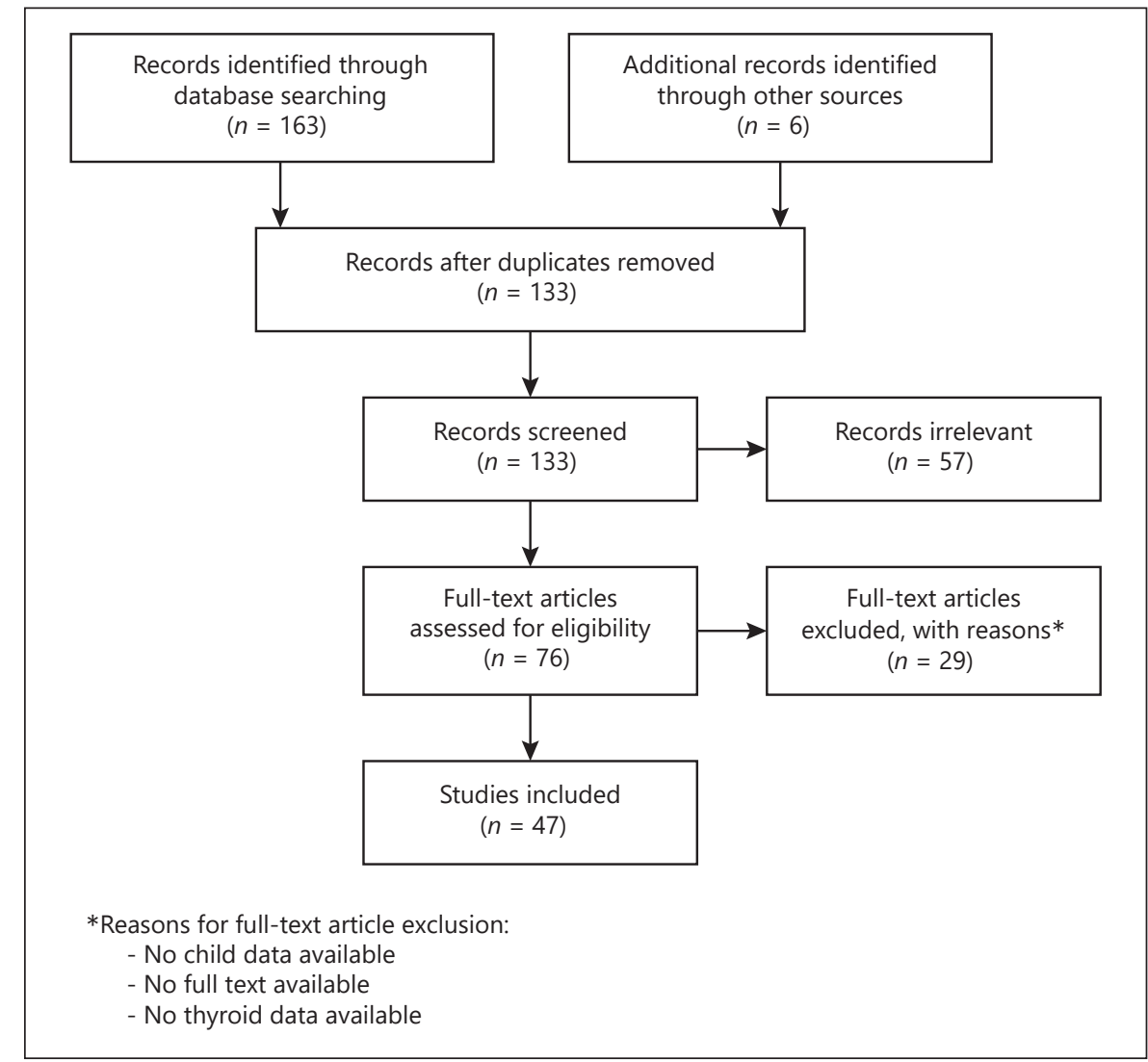

\section{Results}

Question 1: Incidence and Age of Onset of DTC in Children with PHTS

Incidence Studies

Five studies were found describing the incidence of DTC in pediatric patients with PHTS (Table 1). Bubien et al. [10] calculated the cumulative risk for developing DTC in PHTS to be 5\% for patients under 20 years of age. Tan et al. [11] confirmed this cumulative risk in a similar study: of the 105 patients with a PTEN mutation, with an age distribution ranging from 3 to 78 years, 5 developed DTC under the age of 20 years (4.8\%). The results of Riegert-Johnson et al. [12] were concordant: in their cohort of 211 PHTS patients, $4 \%$ developed DTC before the age of 20 , of which the youngest DTC patient was 10 years old. These studies included patients from academic hospitals and beyond in Western countries. In 2 other studies with child-only cohorts, the risk of developing DTC at the pediatric age was estimated to be around $12 \%$, and the risk for developing benign nodular disease (BND) was estimated to be around $50 \%[13,14]$. These studies included the patients from academic hospitals. Nieuwenhuis et al.
Table 1. Studies describing DTC childhood PHTS patients

\begin{tabular}{lrll}
\hline Study & $\begin{array}{l}\text { Risk, } \\
\%\end{array}$ & $\begin{array}{l}\text { Sample } \\
\text { size, } \\
n\end{array}$ & $\begin{array}{l}\text { Youngest age } \\
\text { at diagnosis, } \\
\text { years }\end{array}$ \\
& & & \\
\hline $\begin{array}{l}\text { Adult/child cohort } \\
(10-78 \text { years })^{\mathrm{a}}\end{array}$ & 5 & 140 & 16 \\
$\quad$ Bubien et al. [10] & 4.8 & 105 & not reported \\
$\quad$ Tan et al. [11] & 4 & 211 & 10 \\
$\quad$ Riegert-Johnson et al. [12] & & & \\
$\begin{array}{l}\text { Child/adolescent cohort } \\
\text { (<21 years) }\end{array} \quad 12$ & 34 & 7 \\
$\quad$ Smpokou et al. [14] & 12 & 16 & 6 \\
\hline Plamper et al. [13] & & & \\
\hline
\end{tabular}

${ }^{a}$ Risk analysis for DTC (\%) of the child cohort is shown.

[15] reported that females had a higher risk of developing DTC than males, and this seems to be true for children as well. Ngeow et al. [16] reported that DTC was overrepresented in PHTS patients who carry a mutation in exon 5 of the PTEN gene. Frame shift PTEN mutations were also common amongst PHTS patients diagnosed with DTC, especially in the pediatric age group [17]. 


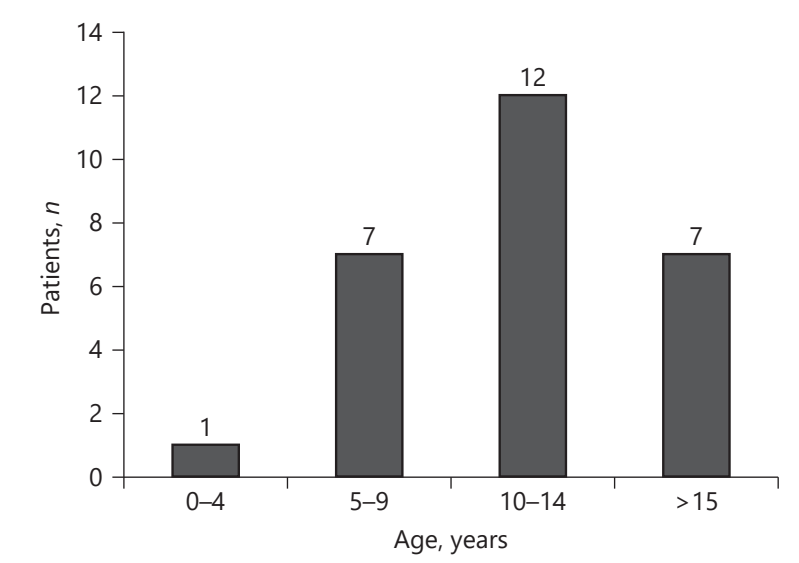

Fig. 2. Age distribution of pediatric patients with PHTS diagnosed with DTC. In total, 27 children ( $<18$ years) with PHTS were identified with DTC. These patients were divided into 4 age categories: $0-4$ years $(n=1), 5-9$ years $(n=7), 10-14$ years $(n=12),>15$ years $(n=7)$.

\section{Cases}

Age at Onset. Fifty-five children with PHTS with DTC and/or BND were identified in the literature. Adding to our 2 cases, 27 out of 57 children had proven DTC, 27 had proven $\mathrm{BND}$, and in a number of cases histology was not reported (several children had both DTC and BND). Six children had thyroiditis (online suppl. Appendix A; see www. karger.com/doi/10.1159/000508872 for all online suppl. material). By combining all cases reported in the literature, the median age at diagnosis of DTC in the pediatric PHTS population was 12 years (range 4-17); the median age for developing BND was also 12 years (range $5-17$ ). The youngest age at which DTC had been diagnosed was 4 years (follicular thyroid carcinoma), with most cases of DTC diagnosed between the ages of 10 and 14 years (Fig. 2).

Mode of Discovery. The way in which DTC and BND had been detected was reported in some, but not in all cases (online suppl. Appendix B). Of the pediatric DTC patients, 7 were identified by physical examination, 4 by thyroid ultrasound, 1 due to observation by an attentive family member, 1 in preoperative evaluation for tonsillectomy, and DTC in 1 patient was found by evaluation of histology results. Of the proven BND patients, 4 were identified by physical examination, 8 by thyroid ultrasound, and 3 by attentive family members.

\section{Question 2: Cancer Type and Behavior}

In $52 \%$ of the cases identified in the literature with reported histology, follicular thyroid carcinoma (FTC) was diagnosed, as compared to a prevalence of $10 \%$ FTC in children without a PTEN mutation [18]. This overrepre-

Thyroid Surveillance in Children with PHTS sentation of FTC in pediatric PHTS patients is in agreement with the recognition of FTC being a major clinical characteristic by the NCCN.

No reports of more aggressive behavior of DTC, defined as increased risk for metastasized disease at diagnosis, recurrence, or increased morbidity or mortality when compared to children with sporadic DTC, could be found. The one study reporting on this topic showed low metastases and recurrence rates in children with PHTS; of the 32 PTEN mutation-positive patients diagnosed with DTC, 2 presented with cervical metastases and 1 with distant metastases at the time of diagnosis. With an average follow-up time of 3.3 years, no recurrences of DTC were identified [19]. The cases identified by our literature study confirmed the relatively mild course of disease; of the 27 DTC cases, only 2 had reported metastatic DTC, and in 2 cases a recurrence was mentioned.

\section{Question 3: Does Early Detection of DTC in Children} with PHTS Improve Outcome?

No evidence was found for children with PHTS. In an extensive expert evaluation using the GRADE system by the International Guideline Harmonization Group, some evidence was found to answer this question in non-PHTS patients [20]. For adults, level A evidence was found that detection of DTC in an early stage may lead to a lower mortality rate and a decreased risk of hypoparathyroidism [20], and level B evidence was found that it leads to lower recurrence rates [20]. In addition, level B evidence was found that lower ${ }^{131} \mathrm{I}^{-}$activity will decrease the risk for second primary malignancies caused by radiation exposure [20]. For children, only level $\mathrm{C}$ evidence could be found that finding DTC in an early stage results in a lower risk for recurrence and lower mortality [20]. Conflicting evidence was found for the effect of diagnosing DTC in an early stage and the risk for morbidity.

\section{Formulating Recommendations with the Current Available Evidence}

Question 1: Incidence of DTC in Children with PHTS

The data suggest that the prevalence of DTC at the pediatric age is approximately $4-12 \%$. In concordance with the recommendations for other low-grade childhood tumors with excellent prognosis (such as Wilms' tumor), surveillance was deemed worthwhile when the risk of disease exceeds $5 \%[8,9]$. Considering the data gathered for this study, it is expected that only in teenage children (>age 10) does the risk for DTC exceed 5\%. 
With these facts in mind, the study team recommends surveillance for DTC in PHTS from the age of 10 years onwards.

\section{Question 2: Does DTC in PHTS Behave Differently} than Sporadic DTC?

Sporadic childhood DTC, although it generally presents with a more advanced disease stage when compared to adults, has a very good prognosis. The 10 -year survival rate for papillary thyroid carcinoma (PTC) varies between 93 and 99\%, and the 10-year survival rate for childhood FTC varies between 96 and 100\% [18, 21, 22].

DTC in children with PHTS might have a different clinical behavior than sporadic DTC since differences in oncogenic pathways involved can lead to differences in cancer phenotype [1]. For this reason, it may be valuable to identify the cancer phenotype (such as disease progression) caused by PTEN protein inactivation, as it may help to understand the behavior of DTC in PHTS patients. In animal models, evidence exists that PTEN mutations are more common in the highly malignant anaplastic thyroid carcinoma and that loss of heterozygosity results in higher levels of invasion and loss of differentiation [21-25]. This may suggest that PTEN mutations potentially contribute towards a thyroid carcinoma type that is more aggressive in nature, and more prone to becoming poorly differentiated $[26,27]$.

No reports could, however, be found on PTEN mutations causing pediatric DTC with a more aggressive phenotype compared to pediatric DTC without a PTEN mutation. In fact, PTEN mutations in sporadic adult DTC were not associated with tumor invasiveness, metastases, and recurrence, and the low metastatic and recurrence rates we found in this review of the literature may even suggest that DTC in pediatric PHTS patients has a possibly milder disease progression [28, 29].

The national expert panel concluded that, due to lack of reports of more aggressive behavior of DTC in PHTS compared to sporadic DTC, data about advantages and disadvantages of surveillance for DTC in other populations such as childhood cancer survivors may be applied, due to the fact that similar principles with regard to surveillance of DTC exist [30].

\section{Question 3: Does Early Detection of DTC in Children} with PHTS Improve Outcome?

If early detection of DTC improved outcome, this would be an argument for an active surveillance program. No evidence for improved outcome was found for PHTS children and for children in the International Guideline Harmonization Group initiative, only level C or conflict- ing evidence was found. Consensus within the study team for the PTEN hamartoma population was therefore reached based on the opinion of the national expert panel.

For children with PHTS, the national expert panel recommends that, although DTC in children has a good prognosis, surveillance for DTC is desirable aiming to detect DTC in an early stage. This may lead to improved disease outcome, mainly due to less complicated surgery. Advanced disease, such as invasive growth and metastases, requires more extensive surgery, thereby increasing the chance of complications such as recurrent laryngeal nerve injury and hypoparathyroidism. Moreover, advanced disease requires a higher cumulative ${ }^{131} \mathrm{I}^{-}$activity which is undesirable in young patients and in patients at risk for secondary malignancies [20].

Based on these arguments, the national expert panel recommends surveillance for DTC in children with PHTS to enable detection of DTC in an early stage.

\section{Cases}

Two cases are presented that illustrate the potential disadvantage of surveillance and that of nonsurveillance.

\section{Case 1: An Example of a Potential Disadvantage of}

Surveillance

A boy, diagnosed with PHTS, received routine thyroid ultrasound at the age of 8 years which revealed multiple thyroid nodules without calcifications. The largest nodule had a diameter of $5 \mathrm{~mm}$, without suspicious cervical lymph nodes. Six months later, a followup ultrasound showed unchanged thyroid nodules and, again, no suspicious lymph nodes. Another 6 months later, a third ultrasound showed multiple hypoechogenic nodules of which the largest was still $5 \mathrm{~mm}$, but now multiple bilateral nonenlarged prominent lymph nodes were seen with several hyperechogenic foci; additionally, enlarged inhomogeneous salivary glands were noted. These prominent lymph nodes were interpreted as suspicious for malignancy among others because of the boy's PHTS. Because the prominent lymph nodes persisted over the following weeks, one node was removed (surgical approach chosen over fine-needle aspiration cytology on surgeon's preference) for pathological examination, which demonstrated a reactive lymph node without signs of malignancy. Despite this reassuring result, later on the boy and his parents reported that they had been very distressed during and after this period. They wondered if all these investigations had really been necessary.

Currently, after a follow-up time of 5 years, the boy is doing well with no palpable nodules. In agreement with the boy and his parents, the future thyroid surveillance strategy consists of annual thyroid ultrasound examination.

Case 2: An Example of a Potential Disadvantage of Not Performing Surveillance

A girl presented at the age of 4 years with Graves' disease. At the age of 5 years, genetic testing was performed for the combina- 
Table 2. Pros and cons of screening for DTC

Arguments for and against DTC surveillance of patients at risk (independently of surveillance modality)

Advantages

Patients at risk whose DTC is detected by surveillance are likely to have it detected at an earlier stage. This may reduce the extent of surgery and/or additional

radioiodine therapy, which could decrease overall morbidity, recurrence as well as morbidity

Patients at risk who are shown not to have a DTC after surveillance benefit by being reassured that they do not have cancer

Disadvantages

There is uncertainty about the benefit of early treatment since most DTC can be cured. There are no randomized studies that demonstrate a clear benefit

of DTC surveillance

Detection of a benign nodule with surveillance (false-positive results for DTC) can lead to repeated ultrasounds, fine-needle aspiration biopsies or thyroid

surgery. These patients may experience unnecessary stress and anxiety in the process of ruling out DTC, as well as inconvenience and complications of unnecessary biopsies or surgery

There is a risk for detection of an indolent DTC which might have a less aggressive natural course which can lead to overtreatment

False-negative results of surveillance may lead to some patients being falsely reassured that they do not have DTC, when in fact they do

Arguments for and against DTC surveillance with neck palpation

Advantages

Quick, inexpensive, and noninvasive

High specificity (96-100\%) for detecting a thyroid nodule that might represent DTC (many true-negative and few false-positive results for nodules)

Disadvantages

Low sensitivity (17-43\%) for detecting a thyroid nodule that might represent DTC (few true-positive and many false-negative results for nodules)

Increase in unnecessary invasive procedures due to false-positive screening results

Detection of DTC at a more advanced stage (compared to thyroid ultrasonography), possibly leading to increased morbidity, recurrence, and mortality rate

Diagnostic value depending on experience of the physician (high-interobserver variation)

Arguments for and against DTC surveillance with thyroid ultrasonography

Advantages

Noninvasive

High sensitivity (approx. 95-100\%) for detecting a thyroid nodule that might represent DTC (many true-positive and few false-negative results for nodules)

High specificity (approx. 95-100\%) for detecting a thyroid nodule that might represent DTC (many true-negative and few false-positive results for nodules)

Detection of DTC at an earlier stage (compared to neck palpation)

Disadvantages

Poor diagnostic value of ultrasound for predicting whether a detected nodule is a DTC: detection of a high number of benign thyroid nodules and indolent DTC

Increase in unnecessary invasive procedures due to false-positive screening results

Diagnostic value depended on experience of the ultrasonographer (high-interobserver variation)

DTC, differentiated thyroid carcinoma. Adapted from Clement et al. [30].

tion of tall stature, hypertelorism, and macrocephaly. A c.517 C>T mutation in the PTEN gene was found, confirming the diagnosis of PHTS.

Because of her young age, with persisting Graves' disease, total thyroidectomy was preferred over radioactive iodine and was performed at the age of 9 years. Ultrasound surveillance for DTC had not been performed. During surgery, the thyroid gland felt "hardened," and regional lymph involvement was suspected, for which several suspicious lymph nodes were removed. Pathological examination of the thyroid gland proved lymphocytic thyroiditis (Graves' disease) and PTC in the left thyroid lobe $(1.4 \mathrm{~cm}$ in diameter) as well as in all of the 18 removed lymph nodes. A therapy of ${ }^{131} \mathrm{I}^{-}(5,516 \mathrm{MBq})$ was administered, and on the postablation scan additional metastatic lymph nodes in the left supraclavicular region and bilateral in level II were seen.

After 6 months, follow-up neck ultrasound revealed multiple enlarged lymph nodes with calcifications, and biopsy again dem- onstrated PTC. Additional lymph node resection was performed, which was complicated by thoracic duct injury necessitating drainage. This was complicated by a wound abscess, requiring surgery.

After recovery, 1 year after the first ${ }^{131} \mathrm{I}^{-}$treatment, a second ${ }^{131} \mathrm{I}^{-}(5,500 \mathrm{MBq})$ treatment was given. Another 6 months later, no suspicious lesions were seen on follow-up ultrasound examination of the neck.

Currently, the girl is under observation by means of annual neck palpation and ultrasound examination, and measurement of the serum thyroglobulin concentration. At the last follow-up, the girl is doing reasonably well. Because of permanent hypothyroidism and hypoparathyroidism, she is treated with thyroxine, calcitriol, and calcium. After this experience, the parents requested active surveillance for any other malignancy that the girl has an increased risk for, regardless of the fact that the girl is not yet fulfilling the starting age for these types of surveillance. 


\section{Discussion}

We summarized the available evidence on the incidence and behavior of DTC and BND and its prognosis in children diagnosed with PHTS. Unfortunately, no studies could be found evaluating long-term DTC surveillance in PHTS patients. Our study confirms that children with PHTS are at increased risk of developing DTC and BND, with an incidence of $5 \%$ from the age of 10 onwards. DTC in PHTS does not seem to behave differently from sporadic DTC. DTC detection at an early stage seems to be beneficial to improve morbidity. The national expert panel recommends surveillance for DTC in children with PHTS from the age of 10 years onwards.

The optimal mode of surveillance for detecting DTC at an early stage is debated. Surveillance for DTC can be done either by neck palpation or by thyroid ultrasound. Thyroid ultrasound is most sensitive but not specific, neck palpation on the other hand has a very low sensitivity and specificity. Both ways of surveillance have their advantages and disadvantages [30].

Recently, a surveillance recommendation has been formulated for childhood cancer survivors at increased risk for DTC [30]. The pros and cons of thyroid surveillance as identified by this study are shown in Table 2 . Due to the fact that behavior of DTC in childhood cancer survivors (CCS) and in PHTS does not seem to differ from sporadic DTC, data on DTC surveillance in CCS were used to form recommendations for DTC surveillance in children with PHTS, based on similar principles. However, although there are similarities between thyroid cancer surveillance in CCS after neck irradiation and children with PHTS, there are also some important differences that must be mentioned. Children with PTEN have a higher prevalence of benign nodular disease than when compared to CCS, which makes the interpretation of the ultrasound images much more difficult. In the irradiated thyroid gland, often a solitary nodule is found. Due to the fact that the interpretation of a thyroid nodule, possibly being malignant, in the thyroid gland of a child with the PHTS is challenging, radiological surveillance should only be performed in centers with high-volume thyroid imaging.

Active surveillance is likely to identify all DTCs; however, this may have disadvantages. The most important argument to advocate active surveillance is that early detection of DTC is associated with better disease outcome [20]. Our case No. 2 is illustrative of the possible natural course of DTC in PHTS with no active thyroid surveillance.
The disadvantage of intensive surveillance with thyroid ultrasound is however the increase in the number of incidental findings, with the inherent risks of unnecessary fine-needle aspirations and even (hemi)thyroidectomies [31]. Additionally, thyroid surgery may inflict unwanted complications. Also, there is currently no evidence that DTC in children with PHTS behaves more aggressively making exhaustive surveillance unnecessary. Case No. 1 is a good example of the clinical and psychological uproar that DTC surveillance may cause, when surveillance results in a false-positive outcome.

By combining the available data in the literature with both its limitations and advantages of surveillance possibilities, and the study team's opinion, consensus was reached to recommend surveillance for DTC in all children with PHTS by means of annual neck palpation and triennial thyroid ultrasound from the age of 10 years onwards. Surveillance from 10 years onwards may be justified by the fact that DTC in PHTS seems to have a relatively mild disease progression and is very rare before the age of 10 years.

Acknowledging its low sensitivity, we recommend yearly neck palpation as surveillance tool, which may be done by the treating physician of the child (this will mostly be a general pediatrician). Despite its low sensitivity (17-43\%), neck palpation has high specificity (96-100\%) and low false-positive rates for detecting thyroid nodules [30]. It is a quick, noninvasive strategy for detecting nodules, without a high risk of finding an incidentaloma requiring additional investigations.

If the initial ultrasound of the thyroid gland, performed in a thyroid or PHTS expertise center, did not reveal any thyroid nodule, we recommend ultrasound of the thyroid gland and neck lymph nodes not more than once every 3 years. This time interval was chosen as this will lead to fewer incidental findings compared to annual surveillance but will detect DTC in an early stage. In case of a thyroid nodule larger than $10 \mathrm{~mm}$, or a smaller nodule with a combination of several suspicious characteristics (solid components, hypoechogenicity, microlobulations or irregular margins, calcifications, and taller-thanwider shape), or when suspicious lymph nodes are detected, fine-needle aspiration cytology is indicated. If a nodule is smaller than $10 \mathrm{~mm}$ without suspicious characteristics, follow-up ultrasound examination 6 months later is indicated to assess whether the nodule has acquired any new features of malignancy. It is essential that an experienced thyroid radiologist performs the ultrasound. Reports upon the use of the ACR TIRADS classification as reporting system in children show contradictory re- 
sults about its adequacy in children [32,33], and no studies exist that evaluate this classification system in children with a germline PTEN mutation. For this reason, no recommendation for using this reporting system can be made.

The strength of our study is that it summarizes all available literature on DTC and BND in children diagnosed with PHTS, and that consensus for recommendation was reached with a national multidisciplinary expert team. Limitations are the lack of studies published on DTC in PTEN mutation-positive children influencing the applicability of our results to the general pediatric PHTS population. The results obtained from the literature-derived cases must be interpreted with caution. The clinical information provided about these cases varied greatly, such as great variability in follow-up time, rendering it impossible to generalize them into a homogeneous sample. The reported risk can be an overestimation due to publication bias, and due to the fact that studies often recruited their patients in academic centers. Moreover, if a child develops DTC this can lead to the diagnosis of PHTS earlier, thereby enriching the childhood PHTS population with DTC cases. On the other hand, the reported risk for developing DTC might be an underestimation. In 3 retrospective studies the investigators based the diagnoses on available clinical information obtained from referring physicians or primary clinical records, in which it was not specifically stated whether all patients were screened for the presence or absence of
DTC. Despite this low level of evidence, by linking incidence and behavior of DTC in PHTS to previously formed recommendations for childhood tumor predisposition syndromes and for CCS at risk for DTC, we tried to augment the evidence underlying this recommendation. These recommendations provide guidance for surveillance of DTC in children with PHTS. However, the importance of individual considerations evaluated by experts in this field and shared decision making with the patient and parents should be emphasized. The present surveillance strategy should be evaluated in time in order to prove its efficacy or be adjusted in case of new upcoming evidence.

\section{Statement of Ethics}

Informed consent was obtained from both patients and their parents for publication.

\section{Disclosure Statement}

The authors have nothing to disclose.

\section{Funding Sources}

This research received no outside support.

\section{References}

1 Pecorino L. Molecular Biology of Cancer: Mechanisms, Targets, and Therapeutics. OUP Oxford; 2012.

2 Tan MH, Mester JL, Ngeow J, Rybicki LA, Orloff MS, Eng C. Lifetime cancer risks in individuals with germline PTEN mutations. Clin Cancer Res. 2012 Jan;18(2):400-7.

3 Daly MB, Pilarski R, Berry M, Buys SS, Farmer M, Friedman S, et al. NCCN Guidelines Insights: Genetic/Familial High-Risk Assessment: Breast and Ovarian, Version 2.2017. J Natl Compr Canc Netw. 2017 Jan;15(1):9-20.

4 Francis GL, Waguespack SG, Bauer AJ, Angelos P, Benvenga S, Cerutti JM, et al.; American Thyroid Association Guidelines Task Force. Management Guidelines for Children with Thyroid Nodules and Differentiated Thyroid Cancer. Thyroid. 2015 Jul;25(7):716-59.

5 Group UC. Guidelines for management of tumour risk in PTEN hamartoma syndrome 2017. Available from: https://www.ukcgg. org/media/10879/pten_management_-cgg_4may2017.pdf.
6 Kets CM, Ten Broeke SW, Bult P, Caanen BA, Hoogerbrugge N, De Hullu JA, et al. De richtlijn PTEN hamartoom tumorsyndroom. Nederlands Tijdschrift voor Oncologie. 2015; 12(4):160-3.

7 Schultz KA, Rednam SP, Kamihara J, Doros L, Achatz MI, Wasserman JD, et al. PTEN, DICER1, FH, and Their Associated Tumor Susceptibility Syndromes: Clinical Features, Genetics, and Surveillance Recommendations in Childhood. Clin Cancer Res. 2017 Jun;23(12):e76-82.

8 Scott RH, Walker L, Olsen OE, Levitt G, Kenney I, Maher E, et al. Surveillance for Wilms tumour in at-risk children: pragmatic recommendations for best practice. Arch Dis Child. 2006 Dec;91(12):995-9.

9 Brodeur GM, Nichols KE, Plon SE, Schiffman JD, Malkin D. Pediatric Cancer Predisposition and Surveillance: An Overview, and a Tribute to Alfred G. Knudson Jr. Clin Cancer Res. 2017 Jun;23(11):e1-5.
10 Bubien V, Bonnet F, Brouste V, Hoppe S, Barouk-Simonet E, David A, et al.; French Cowden Disease Network. High cumulative risks of cancer in patients with PTEN hamartoma tumour syndrome. J Med Genet. 2013 Apr;50(4):255-63.

11 Tan MH, Mester J, Peterson C, Yang Y, Chen JL, Rybicki LA, et al. A clinical scoring system for selection of patients for PTEN mutation testing is proposed on the basis of a prospective study of 3042 probands. Am J Hum Genet. 2011 Jan;88(1):42-56.

12 Riegert-Johnson DL, Gleeson FC, Roberts M, Tholen K, Youngborg L, Bullock M, et al. Cancer and Lhermitte-Duclos disease are common in Cowden syndrome patients. Hered Cancer Clin Pract. 2010 Jun;8(1):6.

13 Plamper M, Schreiner F, Gohlke B, Kionke J, Korsch E, Kirkpatrick J, et al. Thyroid disease in children and adolescents with PTEN hamartoma tumor syndrome (PHTS). Eur J Pediatr. 2018 Mar;177(3):429-35. 
14 Smpokou P, Fox VL, Tan WH. PTEN hamartoma tumour syndrome: early tumour development in children. Arch Dis Child. 2015 Jan; 100(1):34-7.

15 Nieuwenhuis MH, Kets CM, Murphy-Ryan M, Yntema HG, Evans DG, Colas C, et al. Cancer risk and genotype-phenotype correlations in PTEN hamartoma tumor syndrome. Fam Cancer. 2014 Mar;13(1):57-63.

16 Ngeow J, Stanuch K, Mester JL, BarnholtzSloan JS, Eng C. Second malignant neoplasms in patients with Cowden syndrome with underlying germline PTEN mutations. J Clin Oncol. 2014 Jun;32(17):1818-24.

17 Ngeow J, Mester J, Rybicki LA, Ni Y, Milas M, Eng C. Incidence and clinical characteristics of thyroid cancer in prospective series of individuals with Cowden and Cowden-like syndrome characterized by germline PTEN, SDH, or KLLN alterations. J Clin Endocrinol Metab. 2011 Dec;96(12):E2063-71.

18 Hogan AR, Zhuge Y, Perez EA, Koniaris LG, Lew JI, Sola JE. Pediatric thyroid carcinoma: incidence and outcomes in 1753 patients. J Surg Res. 2009 Sep;156(1):167-72.

19 Milas M, Mester J, Metzger R, Shin J, Mitchell J, Berber E, et al. Should patients with Cowden syndrome undergo prophylactic thyroidectomy? Surgery. 2012 Dec;152(6):1201-10.

20 Clement SC, Kremer LC, Links TP, Mulder RL, Ronckers CM, van Eck-Smit BL, et al. Is outcome of differentiated thyroid carcinoma influenced by tumor stage at diagnosis? Cancer Treat Rev. 2015 Jan;41(1):9-16.
21 Kowalski LP, Gonçalves Filho J, Pinto CA, Carvalho AL, de Camargo B. Long-term survival rates in young patients with thyroid carcinoma. Arch Otolaryngol Head Neck Surg. 2003 Jul;129(7):746-9.

22 Sugino K, Nagahama M, Kitagawa W, Shibuya H, Ohkuwa K, Uruno T, et al. Papillary Thyroid Carcinoma in Children and Adolescents: Long-Term Follow-Up and Clinical Characteristics. World J Surg. 2015 Sep;39(9):225965.

23 Burrows N, Babur M, Resch J, Ridsdale S, Mejin M, Rowling EJ, et al. GDC-0941 inhibits metastatic characteristics of thyroid carcinomas by targeting both the phosphoinositide- 3 kinase (PI3K) and hypoxia-inducible factor1a (HIF-1 $\alpha$ ) pathways. J Clin Endocrinol Metab. 2011 Dec;96(12):E1934-43.

24 Champa D, Di Cristofano A. Modeling anaplastic thyroid carcinoma in the mouse. Horm Cancer. 2015 Feb;6(1):37-44.

25 Guigon CJ, Zhao L, Willingham MC, Cheng SY. PTEN deficiency accelerates tumour progression in a mouse model of thyroid cancer. Oncogene. 2009 Jan;28(4):509-17.

26 Liu Z, Hou P, Ji M, Guan H, Studeman K, Jensen $\mathrm{K}$, et al. Highly prevalent genetic alterations in receptor tyrosine kinases and phosphatidylinositol 3-kinase/akt and mitogen-activated protein kinase pathways in anaplastic and follicular thyroid cancers. J Clin Endocrinol Metab. 2008 Aug;93(8):3106-16.

27 Santarpia L, El-Naggar AK, Cote GJ, Myers JN, Sherman SI. Phosphatidylinositol 3-kinase/akt and ras/raf-mitogen-activated protein kinase pathway mutations in anaplastic thyroid cancer. J Clin Endocrinol Metab. 2008 Jan;93(1):278-84.
28 Aydoğan BI, Ersöz CC, Sak SD, Güllü S. The Association between Lymph Node Metastasis and Molecular Markers in Differentiated Thyroid Cancer. Acta Endocrinol (Bucur). 2018 Jan-Mar;14(1):55-65.

29 Kim JS, Bae JS, Kim KH, Ahn CH, Oh SJ, Jeon HM, et al. Clinical Analysis of PTEN, p53 and Her-2/neu Expressions in Thyroid Cancers. Cancer Res Treat. 2001 Oct;33(5):433-7.

30 Clement SC, Kremer LC, Verburg FA, Simmons JH, Goldfarb M, Peeters RP, et al. Balancing the benefits and harms of thyroid cancer surveillance in survivors of Childhood, adolescent and young adult cancer: Recommendations from the international Late Effects of Childhood Cancer Guideline Harmonization Group in collaboration with the PanCareSurFup Consortium. Cancer Treat Rev. 2018 Feb;63:28-39.

31 Lowenstein LM, Basourakos SP, Williams MD, Troncoso P, Gregg JR, Thompson TC, et al. Active surveillance for prostate and thyroid cancers: evolution in clinical paradigms and lessons learned. Nat Rev Clin Oncol. 2019 Mar;16(3):168-84.

32 Lim-Dunham JE, Toslak IE, Reiter MP, Martin B. Assessment of the American College of Radiology Thyroid Imaging Reporting and Data System for Thyroid Nodule Malignancy Risk Stratification in a Pediatric Population. AJR Am J Roentgenol. 2019 Jan;212(1):18894.

33 Richman DM, Benson CB, Doubilet PM, Wassner AJ, Asch E, Cherella CE, et al. Assessment of American College of Radiology Thyroid Imaging Reporting and Data System (TI-RADS) for Pediatric Thyroid Nodules. Radiology. 2020 Feb;294(2):415-20. 